\title{
Size of the dark side of the solar neutrino parameter space
}

\author{
M. C. Gonzalez-Garcia and C. Peña-Garay \\ Instituto de Física Corpuscular - C.S.I.C., Departamento de Física Teòrica, Universitat de València, Edificio Institutos de Paterna, \\ Apt. 2085, 46071 València, Spain
}

(Received 28 February 2000; published 27 June 2000)

\begin{abstract}
We present an analysis of the MSW neutrino oscillation solutions of the solar neutrino problem in the framework of two-neutrino mixing in the enlarged parameter space $\left(\Delta m^{2}, \tan ^{2} \theta\right)$ with $\theta \in(0, \pi / 2)$. Recently, it was pointed out that the allowed region of parameters from a fit to the measured total rates can extend to values $\theta \geqslant \pi / 4$ (the so-called "dark side") when higher confidence levels are allowed. The purpose of this Rapid Communication is to reanalyze the problem, including all the solar neutrino data available, to discuss the dependence on the statistical criteria in the determination of the C.L. of the "dark side" and to extract the corresponding limits on the largest mixing allowed by the data. Our results show that when the SuperKamiokande data on the zenith angle distribution of events and the spectrum information are included, the regions extend more into the second octant.
\end{abstract}

PACS number(s): 26.65.+t, 13.15.+g, 14.60.Pq, 91.35. -x

In a recent work, de Gouvea et al. [1] pointed out that the study of two-active neutrino oscillations in the framework of the Mikheyev-Smirnov-Wolfenstein (MSW) [2] solutions to the solar neutrino deficit as done traditionally on the $\left(\Delta m^{2}, \sin ^{2}(2 \theta)\right)$ parameter space is incomplete since it covers only the range $0 \leqslant \theta \leqslant \pi / 4$ (which they denote as the "light side"). By fitting the data on the total rates measured at chlorine [3], gallium [4,5], and Super-Kamiokande [6] experiments, they claim that the allowed region of parameters extends to the region with $\theta \geqslant \pi / 4$ (denoted as the "dark side") at some reasonable confidence level (C.L.). In fact, to our knowledge, the need of extending the mixing parameter space was first discussed in Ref. [7] where the mixing variable $\tan ^{2} \theta$ was introduced to chart the full mixing range $0 \leqslant \theta \leqslant \pi / 2$.

In this Rapid Communication we reexamine the problem of the extension of the "dark side," that we will denote simply as second octant, of the solar neutrino parameter space after including the effect of all other solar neutrino observables. In particular in this analysis we use all measured total event rates as well as the 825-day SuperKamiokande data on the zenith angle dependence and the recoil electron energy spectrum of the events. We also discuss the dependence on the statistical criteria used in the construction of the allowed regions. Our results are summarized in Tables I and II where we show the maximum allowed values of $\tan ^{2} \theta$ at the 90 and $99 \%$ C.L. when the different observables are included as well as the C.L. for the second octant of the parameter space. We show that when adding the zenith angle and the spectrum information in the analysis, the regions extend more into the second octant. In particular for the large mixing angle (LMA) solution, this behavior is mainly driven by the data on the zenith angular dependence since the best-fit point for the zenith angle distribution is in the second octant as pointed out in Ref. [8]. For details on the data and statistical analysis employed in this study we refer to our detailed work on Ref. [9] and references therein.
Let us first recall that in the framework of two massive neutrinos, the weak eigenstates $\left(\nu_{e}\right.$ and $\nu_{x}$ for the solar neutrino problem) can be written as a linear combination of the mass eigenstates $\nu_{1}$ and $\nu_{2}$

$$
\begin{aligned}
& \nu_{e}=\cos \theta \nu_{1}+\sin \theta \nu_{2}, \\
& \nu_{x}=-\sin \theta \nu_{1}+\cos \theta \nu_{2},
\end{aligned}
$$

where $\theta$ is referred to as the mixing angle in vacuum. The mass-squared difference is defined as $\Delta m^{2}=m_{2}^{2}-m_{1}^{2}$. For the solar neutrino problem $\nu_{x}$ can label either an active neutrino $x=\mu, \tau$ or a sterile neutrino. In what follows we restrict our discussion to oscillations into active neutrinos. For oscillations into sterile neutrinos large mixing solutions are not allowed [9].

By inspection of the symmetry properties of Eq. (1) one sees that the full parameter space can be exhausted by using the mass-squared difference $\Delta m^{2}$ always positive and the

TABLE I. $\chi_{\min }^{2}$ and its g.o.f. (goodness of the fit), maximum allowed values of $\tan ^{2}(\theta)$ at 90 (99) \% C.L. and the confidence level at which $\theta \geqslant \pi / 2$ is allowed in the LMA region for the different set of observables and the two statistical criteria discussed in the

\begin{tabular}{|c|c|c|c|c|}
\hline LMA & $\begin{array}{l}\chi_{\min , L M A}^{2} / \text { d.o.f. } \\
\text { g.o.f. }\end{array}$ & Crit. & $\tan ^{2}(\theta)_{\max }$ & $\begin{array}{l}\text { C.L. (\%) } \\
2^{\text {nd }} \text { Octant }\end{array}$ \\
\hline \multirow[t]{2}{*}{ rates } & $2.9 / 1$ & $\mathrm{C} 1$ & $0.47 \quad(0.76)$ & 99.6 \\
\hline & $91 \%$ & $\mathrm{C} 2$ & $0.62(1.35)$ & 98.7 \\
\hline rates & $7.0 / 6$ & $\mathrm{C} 1$ & $0.62(1.01)$ & 99.0 \\
\hline +zenith & $68 \%$ & $\mathrm{C} 2$ & $0.69(1.15)$ & 98.2 \\
\hline rates & $22.1 / 18$ & $\mathrm{C} 1$ & $0.55(0.92)$ & 99.1 \\
\hline+ spectrum & $77 \%$ & $\mathrm{C} 2$ & $0.55 \quad(0.92)$ & 99.1 \\
\hline \multirow[t]{2}{*}{ global } & $27.0 / 23$ & $\mathrm{C} 1$ & $0.69(1.18)$ & 98.2 \\
\hline & $74 \%$ & $\mathrm{C} 2$ & 0.69 (1.18) & 98.2 \\
\hline
\end{tabular}
text. 
TABLE II. $\chi_{\min }^{2}$ and its g.o.f. (goodness of the fit), maximum allowed values of $\tan ^{2}(\theta)$ at 90 (99)\% C.L. and the confidence level at which $\theta \geqslant \pi / 2$ is allowed in the LOW region for the different set of observables and the two statistical criteria discussed in the text.

\begin{tabular}{lcccc}
\hline \hline LOW & $\begin{array}{c}\chi_{\min , \text { LOW }}^{2} / \text { d.o.f. } \\
\text { g.o.f. }\end{array}$ & Crit. & $\tan ^{2}(\theta)_{\max }$ & $\begin{array}{c}\text { C.L. }(\%) \\
2^{\text {nd }} \text { Octant }\end{array}$ \\
\hline \multirow{2}{*}{ rates } & $7.4 / 1$ & $\mathrm{C} 1$ & $-(0.87)$ & 99.6 \\
& $99 \%$ & $\mathrm{C} 2$ & $1.37(1.78)$ & 82.6 \\
rates & $12.7 / 6$ & $\mathrm{C} 1$ & $-(0.94)$ & 99.1 \\
+zenith & $95 \%$ & $\mathrm{C} 2$ & $1.35(1.77)$ & 75.5 \\
rates & $26.7 / 18$ & $\mathrm{C} 1$ & $-(1.29)$ & 98.1 \\
+ spectrum & $92 \%$ & $\mathrm{C} 2$ & $1.23(1.69)$ & 84.3 \\
global & $32 . / 23$ & $\mathrm{C} 1$ & $-(1.24)$ & 98.3 \\
& $90 \%$ & $\mathrm{C} 2$ & $1.30(1.74)$ & 79.6 \\
\hline \hline
\end{tabular}

mixing angle in the interval $0 \leqslant \theta \leqslant \pi / 2$. In the case of vacuum oscillations, moreover, the transition probabilities as directly derived from Eq. (1) can be written in terms of $\sin ^{2}(2 \theta)$ and therefore they are symmetric under the change $\theta \rightarrow \theta-\pi / 2$ so each allowed value of $\sin ^{2}(2 \theta)$ corresponds to two allowed values of $\theta$.

On the other hand, in the case of the MSW solutions the transition probability takes the form

$$
P_{e e}=P_{e 1}^{S u n} P_{1 e}^{\text {Earth }}+P_{e 2}^{\text {Sun }} P_{2 e}^{\text {Earth }}
$$

where $P_{e 1}^{S u n}$ is the probability that a solar neutrino, that is created as $\nu_{e}$, leaves the Sun as a mass eigenstate $\nu_{1}$, and $P_{1 e}^{E a r t h}$ is the probability that a neutrino which enters the Earth as $\nu_{1}$ arrives at the detector as $\nu_{e}$ [10]. Similar definitions apply to $P_{e 2}^{\text {Sun }}$ and $P_{2 e}^{\text {Earth }}$. For $P_{i e}^{\text {Earth }}$ we integrate numerically the evolution equation in matter using the Earth density profile given in the preliminary reference earth model (PREM) [11].

The quantity $P_{e 1}^{S u n}$ is given, after discarding the oscillation terms, as

$$
P_{e 1}^{S u n}=1-P_{e 2}^{S u n}=\frac{1}{2}+\left(\frac{1}{2}-P_{L Z}\right) \cos \left(2 \theta_{m, 0}\right)
$$

where $P_{L Z}$ denotes the standard Landau-Zener probability [12] and $\theta_{m, 0}$ is the mixing angle in matter at the neutrino production point:

$$
\begin{aligned}
\cos \left(2 \theta_{m, 0}\right) & =\frac{\Delta m^{2} \cos (2 \theta)-A_{0}}{\sqrt{\left(\Delta m^{2} \cos (2 \theta)-A_{0}\right)^{2}+\left(\Delta m^{2} \sin (2 \theta)\right)^{2}}} \\
P_{L Z} & =\frac{\exp \left[-\gamma \sin ^{2} \theta\right]-\exp [-\gamma]}{1-\exp [-\gamma]}, \\
\gamma & =\pi \frac{\Delta m^{2}}{E}\left(\left.\frac{d \ln N_{e}(r)}{d r}\right|_{r=r_{\text {res }}}\right)^{-1},
\end{aligned}
$$

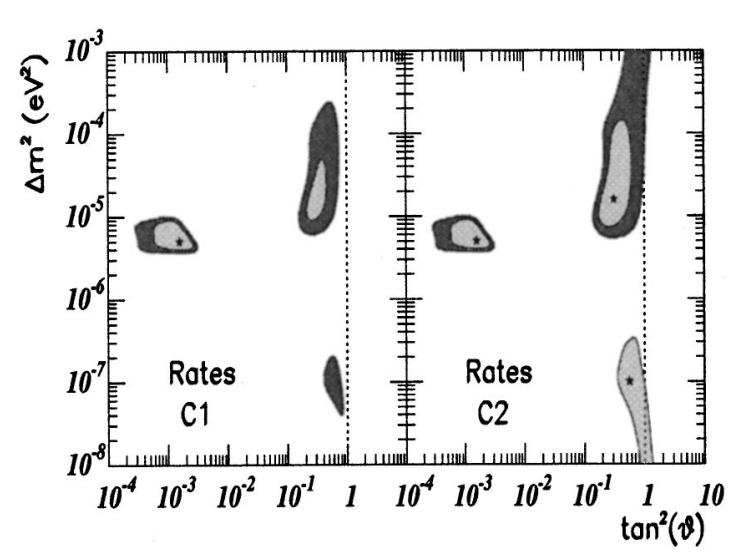

FIG. 1. Allowed regions in $\Delta m^{2}$ and $\tan ^{2} \theta$ from the measurements of the total event rates at chlorine, gallium, and SuperKamiokande (825-day data sample) in the two different statistical criteria. The darker (lighter) areas indicate the 99\% (90\%) C.L. regions. The best-fit points used to define the regions are indicated by a star.

with $A_{0}=2 \sqrt{2} G_{F} E N_{e}\left(r_{0}\right)$ where $N_{e}\left(r_{0}\right)$ is the number density of electrons in the production point, $E$ is the neutrino energy, $G_{F}$ is the Fermi constant, and $r_{r e s}$ is the resonant point $\left(\Delta m^{2} \cos 2 \theta=A_{r e s}\right)$. This probability is clearly not invariant under the change $\theta \rightarrow \pi / 2-\theta$ as resonant transitions are only possible for values of $\theta$ smaller than $\pi / 4$ as seen in Eq. (4) which considerably suppresses the transitions for $\theta$ $>\pi / 4$. For this reason most of the earlier papers on the MSW effect that considered the two-neutrino mixing case used $\left(\Delta m^{2}, \sin ^{2} 2 \theta\right)$ as parameters in the fitting procedure [13] with $\theta \in[0, \pi / 4]$. In some cases the parameter space was represented as $\left(\sin ^{2} 2 \theta\right) /(\cos 2 \theta)[14,15]$ but still assuming $\theta$ to be in the first octant.

Due to the fact that this choice does not exhaust the full parameter space once matter effects are included, other representations have been used to show the enlarged space particularly in the framework of three-neutrino $[7,16]$ and fourneutrino oscillations [8]. In this way two suggestions to parametrize the mixing angle have been made in the literature: $\sin ^{2} \theta$ and $\tan ^{2} \theta$ with $\theta \in[0, \pi / 2]$. For the sake of comparison with the analysis in Ref. [1] we choose to show our results in terms of $\tan ^{2} \theta$.

We now turn to our results. We first determine the allowed range of oscillation parameters using only the total event rates of the chlorine, gallium, and Super-Kamiokande experiments. We have not included in our analysis the Kamiokande data [17] as they are well in agreement with the results from the Super-Kamiokande experiment and the precision of this last one is much higher. For the gallium experiments we have used the weighted average of the results from GALLEX [4] and SAGE [5] detectors. Our results are shown in Fig. 1 where we show the allowed regions. We choose to show our allowed regions for the "conventional" 90 and $99 \%$ C.L. It is obvious that increasing the allowed C.L. would lead to larger regions. The C.L. for the second octant and the maximum values of $\tan ^{2} \theta$ for which the LMA and low mass (LOW) solutions are allowed at those C.L.'s can be found in Tables I and II. Furthermore, in Fig. 2 we show 


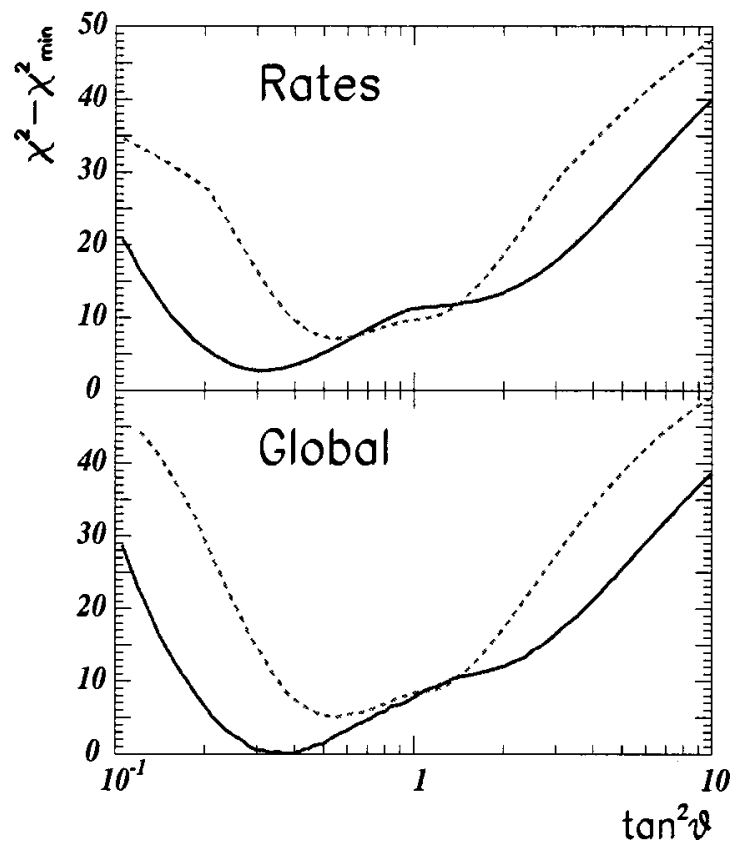

FIG. 2. $\chi_{\text {min }}^{2}\left(\tan ^{2} \theta\right)-\chi_{\text {min }}^{2}$ in the LMA (full lines) and LOW (dashed lines) regions as a function of $\tan ^{2} \theta$, coming from the fit to the total rates (upper curves) and from the global data analysis (lower curves).

the value of $\chi_{\min }^{2}\left(\tan ^{2} \theta\right)-\chi_{\min }^{2}$ in the LMA and LOW regions [where $\chi_{\min }^{2}\left(\tan ^{2} \theta\right)$ is minimized in $\Delta m^{2}$ ] as a fuction of $\tan ^{2} \theta$. From the figure it is possible to extract the maximum allowed values of $\tan ^{2} \theta$ at any other C.L. For instance, taking the global analysis in the LMA region from the figure we see that $\tan ^{2} \theta=3\left(\theta=60^{\circ}\right)$ is possible only for $\Delta \chi^{2}$ $>16.6$ which corresponds to 99.98 for 2 d.o.f. $(3.65 \sigma)$. We present our results according to two different statistical criteria used in the literature in the definition of the allowed parameters. The use of each criterion depends on the physics scenario to which the result of the analysis is to be applied.

Criterion $1(\mathrm{C} 1)$ : The regions at certain C.L. are defined in terms of shifts of the $\chi^{2}$ function for 2-d.o.f., $\Delta \chi^{2}$ $=4.6(9.2)$ at 90 (99) \% C.L., with respect to the global minimum in the full plane. This criterion is used, for instance, in Refs. $[1,6,18,9,8,19]$. It is applicable when no region of the parameter space small mixing angle (SMA), LMA, or LOW is a priori assumed to be the right one.

Criterion 2 (C2): The regions at certain C.L. are defined in terms of shifts of the $\chi^{2}$ function for 2-d.o.f. with respect to the local minimum in the corresponding region. This criterion holds when assuming that a given solution, SMA, LMA, or LOW, is the valid one. It clearly yields less restrictive limits. This criterion is used, for instance, in Refs. [20,8]. In the figures we mark with a star the location of the minima used to define the contours in each case. For instance, in Fig. 1 when using criterion 1 , the contours are defined with respect to minimum $\chi_{\min }^{2}=0.37 / 1$ d.o.f. which is obtained in the small mixing angle solution. For criterion 2 we define each region with respect to its local minimum whose value and the corresponding goodness of the fit is given in Tables I and II, respectively. Notice that the relatively bad goodness

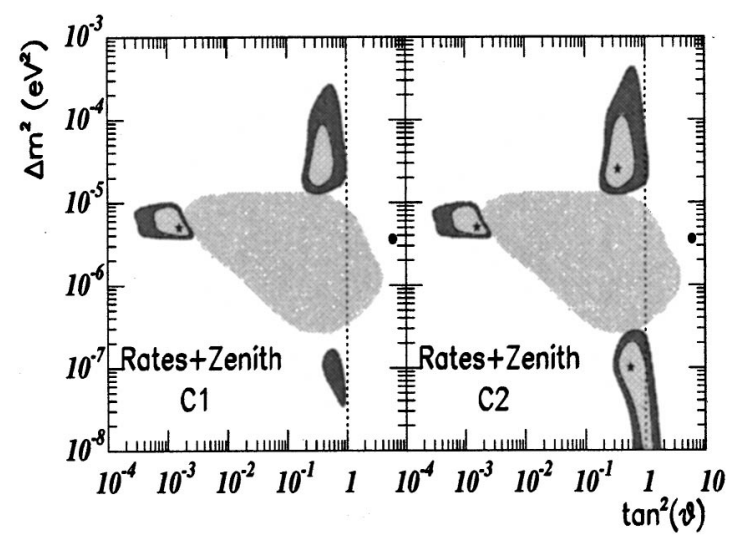

FIG. 3. Same as Fig. 1 but including also the data on the zenith angle distribution observed in Super-Kamiokande. The shadowed area represents the region excluded by the zenith angle distribution data at $99 \%$ C.L. The location of minimum of the $\chi^{2}$ function for the zenith angle data is marked with a point.

of the fit for the LMA and LOW regions from the analysis of the rates only is the reason why for example in Fig. 1 the LMA and LOW regions are quite different using $\mathrm{C} 1$ and $\mathrm{C} 2$.

As seen in Fig. 1 and Tables I and II when using criterion 1 , we do not find any solution in the second octant at $99 \%$ C.L. from the analysis of the rates only. One must increase the C.L. to 99.6 (99.6) for the LMA (LOW) region to extend into values $\tan ^{2} \theta>1$. This is in agreement, for instance, with the results of Ref. [16]. In Ref. [1] they find some small allowed region in the second octant for the LOW solution at the $99 \%$ C.L. We have traced the origin of this small discrepancy to their use of the exponential approximation for the electron number density in the Sun. In our calculation we use the solar neutrino fluxes from Ref. [21] and the new numerical parametrization of the Sun density as given by Bahcall [22]. We have explicitly verified that if using the exponential approximation in our calculation the allowed LOW region extends into the second octant at 99\% C.L.

The situation is changed when the regions are defined according to criterion 2, as seen in Fig. 1 and Tables I and II. In particular both the LMA and LOW regions overlap at 99\% C.L. because the value of $\chi^{2}$ in between the two regions is below the $99 \%$ C.L. defined with respect to the local LOW minimum (for this reason the LOW region in the figure is only shown at the $90 \%$ C.L.). Notice also that in our representation we have chosen to cut the parameter space at $\Delta m^{2}>10^{-8} \mathrm{eV}^{2}$. Recently, in Ref. [23] it has been pointed out that matter effects may be relevant for lower values of $\Delta m^{2}$. One must notice, however, that for such lower mass values the simple analytic expressions in Eqs. (3) and (4) start losing validity $[15,16,24]$.

In Figs. 3 and 4 we show the allowed regions when either the data on the zenith angular dependence or the recoil electron energy spectrum are combined with the results from the total rates. The corresponding values of the absolute minimum of the $\chi^{2}$ function for the combination of rates plus zenith angular dependence data (rates plus recoil electron energy spectrum) are $\chi_{\min }^{2}=5.9 / 3$ d.o.f. $(22.1 / 15$ d.o.f.) which are obtained for the SMA (LMA) solutions and are used in the construction of the allowed regions for criterion 


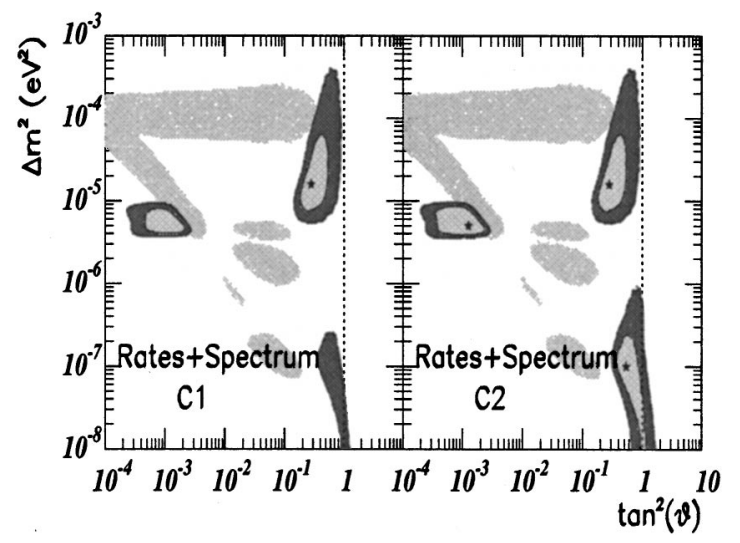

FIG. 4. Same as Fig. 1 but including also the data on the recoil electron energy spectrum observed in Super-Kamiokande. The shadowed area represents the region excluded by spectrum data at 99\% C.L.

1. In the figures we also show the corresponding excluded regions at $99 \%$ C.L. by the new observables.

As seen in Fig. 3 and in the tables the inclusion of the data on the zenith angular dependence of the Super-Kamiokande events leads, in general, to an increase in the maximum allowed values of $\tan ^{2} \theta$ and a better C.L. for the second octant for both LMA and LOW regions. This is due to the fact that the best-fit point for the angular distribution is obtained in the second octant $\left[\Delta m_{21}^{2}=3.7 \times 10^{-6} \mathrm{eV}^{2}, \tan ^{2}(\theta)=5.9\right.$ with $\left.\chi_{\text {min,zen }}^{2}=1.5\right]$ as shown in Fig. 3 [8].

This fact has been obviated in past analysis in the twoneutrino oscillations and it must be taken into account to do properly the $\chi^{2}$ analysis when the data on zenith dependence are included. In this respect, one must notice, for instance, that in their preliminary analysis on the zenith angle dependence [6] Super-Kamiokande obtains the minimum $\chi^{2}$ at the boundary of their "cut" mixing parameter space $\sin ^{2}(2 \theta)$ $=1$. In this way, their $\chi_{\min }^{2}$ is higher than the "true" minimum which is missed. Although, at present, the difference in the excluded region defined with respect to the "true", or with respect to the "cut" minimum is small, this can be of further importance when more data are accumulated.

We have also explicitly verified that the results on the zenith angle excluded region as well as the best-fit point position are very mildly dependent on the exact profile of the Earth. Very similar results can be obtained by using the analytical expressions valid for the two-step Earth density profile [10].

The effect of the inclusion of the recoil electron energy spectrum is shown in Fig. 4. In this case when using criterion 1 we also find an increase in the maximum allowed values of $\tan ^{2} \theta$ and a better C.L. for the second octant for both LMA and LOW regions as compared to the results for the analysis of the rates only. However, this is not the case when using criterion 2 as can be seen in the tables. The allowed regions from the global analysis are displayed in Fig. 5. We see from the figure that at $99 \%$ C.L. both the LMA and the LOW regions extend into the second octant when using any of the two statistical criteria. In Fig. 2 we show the value of $\chi_{\text {min }}^{2}\left(\tan ^{2} \theta\right)-\chi_{\text {min }}^{2}$ in the LMA and LOW regions [where

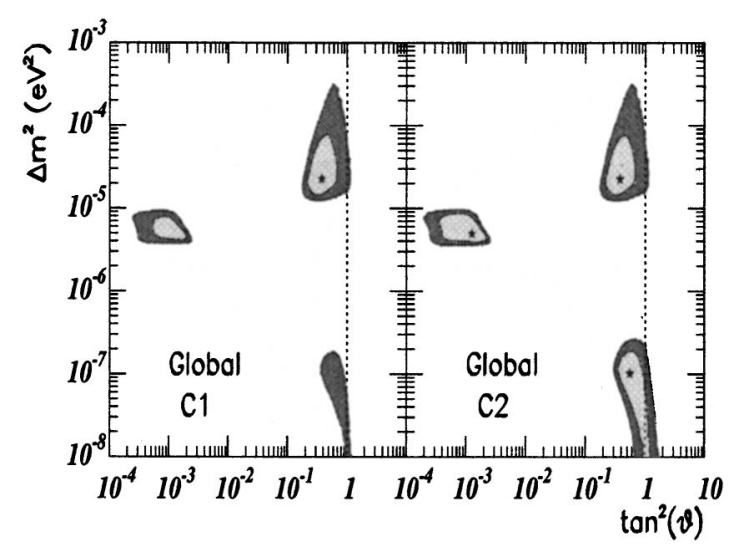

FIG. 5. Same as Fig. 1 for a global fit to the solar neutrino data.

$\chi_{\text {min }}^{2}\left(\tan ^{2} \theta\right)$ is minimized in $\left.\Delta m^{2}\right]$ as a function of $\tan ^{2} \theta$ coming from the fit to the total rates (for which we recall that $\chi_{\min }^{2}=0.37$ ) and to the global data set (for which $\chi_{\min }^{2}$ $\left.=\chi_{\min , L M A}^{2}=27.0\right)$. As seen in the figure the inclusion of the new observables leads to a small shift in the position of the local best-fit points towards slightly larger values of $\tan ^{2} \theta$. However, as discussed before, this does not always translate into an increase on the maximum allowed value of $\tan ^{2} \theta$ as the minimum also becomes deeper.

To summarize, in this paper we have studied the extension of the second octant of the solar neutrino parameter space after including in the analysis all measured total event rates as well as all the 825-day Super-Kamiokande data on the zenith angle dependence and the recoil electron energy spectrum of the events. We also have discussed the dependence of the results on the statistical criterion used in the construction of the allowed regions. Our results are summarized in Tables I and II where we show the maximum allowed values of the $\tan \theta$ at 90 and $99 \%$ C.L. when the different observables are included as well as the C.L. for the second octant of the parameter space. We have shown that when the zenith angle and the spectrum information is included the regions extend more into the second octant. For the LMA this behavior is mainly driven by the data on the zenith angular dependence since the best-fit point for the zenith angle distribution is in the second octant.

Finally, we just comment that the existence of solutions to the solar neutrino problem for $\theta>\pi / 4$ is not only of academic interest to the extent that in general they are perfectly allowed by the models of neutrino masses [25]. A particularly interesting recent example of a predictive model can be found in Ref. [26] where, despite the predictivity of the model, solutions in both octants are equally possible. Furthermore, in the context of three-neutrino mixing, the determination of the sign of $\Delta m^{2}$ of the LMA solution can be of relevance in the exact determination of the $C P$-violating phase [27].

We thank Carlo Giunti, H. Murayama, and J. W. F. Valle for discussions. We are especially indebted to J. Bahcall for providing us with the latest parametrization of the Sun matter density. This work was supported by DGICYT under grants PB98-0693 and PB97-1261, and by the TMR network grant ERBFMRXCT960090 of the European Union. 
[1] Andre de Gouvea, Alexander Friedland, and Hitoshi Murayama, hep-ph/0002064.

[2] S.P. Mikheyev and A.Yu. Smirnov, Yad. Fiz. 42, 1441 (1985) [Sov. J. Nucl. Phys. 42, 913 (1985)]; L. Wolfenstein, Phys. Rev. D 17, 2369 (1978).

[3] B.T. Cleveland et al., Astrophys. J. 496, 505 (1998).

[4] T. Kirsten, talk at the 6th International Workshop on Topics in Astroparticle and Underground Physics, TAUP99, Paris, 1999.

[5] SAGE Collaboration, J.N. Abdurashitov et al., Phys. Rev. C 60, 055801 (1999).

[6] Y. Suzuki, talk at the XIX International Symposium on Lepton and Photon Interactions at High Energies, Stanford University, 1999; M. Nakahata, talk at the 6th International Workshop on Topics in Astroparticle and Underground Physics, TAUP99, Paris, 1999.

[7] G.L. Fogli, E. Lisi, and D. Montanino, Phys. Rev. D 54, 2048 (1996).

[8] C. Giunti, M.C. Gonzalez-Garcia, and C. Peña-Garay, Phys Rev. D 62, 013005 (2000).

[9] M.C. Gonzalez-Garcia, P.C. de Holanda, C. Peña-Garay, and J.W.F. Valle, hep-ph/9906469.

[10] S.T. Petcov, Phys. Lett. B 434, 321 (1998); E.Kh. Akhmedov, Nucl. Phys. B538, 25 (1999).

[11] A.M. Dziewonski and D.L. Anderson, Phys. Earth Planet. Inter. 25, 297 (1981).

[12] S.T. Petcov, Phys. Lett. B 200, 373 (1988); P.I. Krastev and S.T. Petcov, ibid. 207, 64 (1988).

[13] S.P. Mikheyev and A.Yu. Smirnov, Usp. Fiz. Nauk 153, 3 (1987) [Sov. Phys. Usp. 30, 759 (1987)]; J. Bouchez et al., Z. Phys. C 32, 499 (1986); S.P. Rosen and J.M. Gelb, Phys. Rev.
D 34, 969 (1986); W.C. Haxton ibid. 35, 2352 (1987); V. Barger et al., ibid. 34, 980 (1986).

[14] S.J. Parke and T.P. Walker, Phys. Rev. Lett. 57, 2322 (1986); G.L. Fogli, E. Lisi, and D. Montanino, Phys. Rev. D 49, 3626 (1994).

[15] A.J. Baltz and J. Weneser, Phys. Rev. D 37, 3364 (1988).

[16] G.L. Fogli, E. Lisi, D. Montanino, and A. Palazzo, Phys. Rev. D 62, 013002 (2000).

[17] Kamiokande Collaboration, Y. Fukuda et al., Phys. Rev. Lett. 77, 1683 (1996).

[18] J.N. Bahcall, P.I. Krastev, and A.Yu. Smirnov, Phys. Rev. D 58, 096016 (1998).

[19] N. Hata and P. Langacker, Phys. Rev. D 50, 632 (1994); 56, 6107 (1997).

[20] J.N. Bahcall, P.I. Krastev, and A.Yu. Smirnov, Phys. Lett. B 477, 401 (2000).

[21] J.N. Bahcall, S. Basu, and M.H. Pinsonneault, Phys. Lett. B 433, 1 (1998).

[22] http://www.sns.ias.edu/ ${ }^{\sim}$ jnb/SNdata/Export/BP2000; J.N. Bahcall, S. Basu, and M.H. Pinsonneault, Astrophys. J. 529, 1084 (2000).

[23] A. Friedland, hep-ph/0002063.

[24] P.I. Krastev and S.T. Petcov, Phys. Lett. B 299, 99 (1993); Nucl. Phys. B449, 605 (1995).

[25] For a recent review see, for instance, J.W.F. Valle, hep-ph/9907222, and references therein.

[26] J.C. Romao, M.A. Diaz, M. Hirsch, W. Porod, and J.W.F. Valle, Phys. Rev. D 61, 071703 (2000).

[27] For a recent discussion see A. Cervera et al., hep-ph/0002108, and references therein. 